\title{
预填充支撑体制备介孔硅MCM-48 分子篮膜
}

\author{
徐德刊，范益群* \\ 南京工业大学化学化工学院材料化学工程国家重点实验室, 南京 210009 \\ *E-mail: yiqunfan@njut.edu.cn \\ 收稿日期: 2009-06-30; 接受日期: 2009-07-27 \\ 国家自然科学基金(批准号: 20776067)资助项目
}

\begin{abstract}
摘要 利用水溶液填充平均孔径为 $0.2 \mu \mathrm{m}$ 的 $\mathrm{ZrO}_{2}$ 管式非对称支撑体, 水热合成法制备得到了 高通量的 MCM-48 分子篎膜. 首先将支撑体浸泡在预先制备的具有一定 $\mathrm{pH}$ 值的水溶液中, 负压 抽吸以排除支撑体中的气体, 然后放入溶胶中水热合成, 经乙醇/盐酸溶液 $(\mathrm{EtOH} / \mathrm{HCl})$ 萃取干燥 后得到 MCM-48 分子篎膜. 单组分气体 $\left(\mathrm{N}_{2}, \mathrm{H}_{2}\right)$ 渗透实验表明, 利用水溶液填充支撑体与干燥支 撑体相比, 可以明显改善 MCM-48 分子篎成膜质量. 通过二次合成可以得到高通量、完整的 MCM-48 分子䈨膜, 常温、跨膜压差为 $0.05 \mathrm{MPa}$ 时 $\mathrm{N}_{2}$ 渗透系数为 $5.66 \times 10^{-7} \mathrm{~mol} /\left(\mathrm{Pa} \cdot \mathrm{s} \cdot \mathrm{m}^{2}\right), \mathrm{H}_{2} / \mathrm{N}_{2}$ 分离因子可达到 3.47 .
\end{abstract}

关键词

MCM-48 分子笠膜 液体填充
1992 年, Mobil 公司的科学家 ${ }^{[1,2]}$ 报道 M41s 介孔 分子篎的合成方法, 其中 MCM-48 分子篎为 Ia3d 立 方结构, 有两条相互独立的三维孔道体系且满足最 小面螺旋结构 ${ }^{[3]}$, 孔道结构规整, 孔径分布窄, 孔径 2 10 nm 可调 ${ }^{[4]}$. 利用 MCM-48 制备而成的分子篮膜 孔道垂直于支撑体表面, 有利于物质的传递, 是一种 理想的分离材料.

Nishiyama 等人 ${ }^{[5]}$ 首先在多孔不锈钢支撑体上成 功地制备了 MCM-48 膜, 随后又在多孔氧化铝上利用 浸涂法和水热合成法制备 MCM-48 膜并采用气体渗透 实验进行表征 ${ }^{6]}$, 结果表明 $\mathrm{N}_{2}, \mathrm{H}_{2}$ 等气体在室温下通 过 MCM-48 膜受努森流扩散控制. 在 MCM-48 膜的制 备过程中支撑体起着重要的作用, 不同的课题组分别 用不同孔径的支撑体制备 MCM-48 膜 ${ }^{[7 \sim 9]}$, 发现采用 平均孔径 $0.2 \mu \mathrm{m}$ 的支撑体可以制备比较完整的 MCM-48 分离膜. 值得注意的是, Santamaria 等人 ${ }^{[8]}$ 采 用平均孔径为 $0.2 \mu \mathrm{m}$ 的 $\alpha-\mathrm{Al}_{2} \mathrm{O}_{3}$ 非对称管式支撑体制 备 MCM-48 膜, 增大了分离膜的气体渗透系数, 而
$\mathrm{N}_{2} / \mathrm{H}_{2}$ 分离因子未见报道. 最近, Vadim 等人 ${ }^{[10]}$ 在 $\alpha-\mathrm{Al}_{2} \mathrm{O}_{3}$ 非对称支撑体上采用包裹支撑体大孔侧的方法制备得 到了高通量(约 $2.5 \times 10^{-7} \mathrm{~mol} /\left(\mathrm{Pa} \cdot \mathrm{s} \cdot \mathrm{m}^{2}\right)$ )、较完整的 MCM48 膜, 并用 $\mathrm{CO}_{2} / \mathrm{N}_{2}$ 单组分气体渗透实验表征其分离 性能.

Kim 等人 ${ }^{[11]}$ 首先用 PVA 填充支撑体制备了有序 介孔硅薄膜, 实验结果表明液体填充可以防止溶胶 向支撑体内部的渗透, 同时可以减小支撑体表面粗 楉度从而有利于薄膜的制备. Wang 等人 ${ }^{[12,13]}$ 采用硅 溶胶预填充大孔 $\alpha-\mathrm{Al}_{2} \mathrm{O}_{3}$ 支撑体成功的制备得到了 MCM-48 膜, 虽然利用大孔支撑体, 但是其气体渗透 系数并没有明显的提高 ${ }^{[13]}$, 可能是由于硅溶胶填充 在大孔支撑体内, 水热合成过程中形成固体氧化硅 从而增大了气体渗透阻力. 为了得到高通量的 MCM-48 膜, 将填充物有效的去除也是一个非常关 键问题. Kim 等人在制备薄膜后利用炦烧法除去 PVA, 在高温炦烧法过程中, 不但会影响 MCM-48 分子笁 的晶格结构 ${ }^{[8,9,14]}$, 同时会由于支撑体与膜层的热膨

引用格式: Xu D K, Fan Y Q. Mesoporous Si-MCM-48 membrane prepared by pore-filling method. Sci China Tech Sci, 2010, 53: 1064-1068, doi: 10.1007/s11431-009-0365-0 
胀系数的不同容易导致膜表面开裂.

本文提出采用水溶液作为填充物预填充支撑体, 控制水溶液的 $\mathrm{pH}$ 值与 MCM-48 溶胶一致, 这既可以 防止干燥支撑体在溶胶中的毛细吸浆作用, 减小溶 胶向支撑体内部的渗透, 又不会影响 MCM-48 分子 篮膜的水热合成, 同时不用考虑填充物的去除问题. 采用 XRD, BET, SEM 对 MCM-48 材料及膜进行表征, 探讨其合成机理.

\section{1 实验部分}

将支撑体浸泡在预先制备的 $\mathrm{pH}$ 为 11.8 的 $\mathrm{NaOH}$ 水溶液中, 负压(绝对压力 $0.05 \mathrm{MPa}$ )抽吸 $2 \mathrm{~h}$ 以排除 支撑体中的气体. 溶胶的制备同文献[14], 按照摩尔 比为 TEOS: CTAB : NaOH : $\mathrm{H}_{2} \mathrm{O}=1.0: 0.48: 0.46$ $: 56$, 首先将 $\mathrm{NaOH}$ 和 $\mathrm{CTAB}$ 溶于去离子水中磁力摚 拌, 溶解后控制一定的温度滴入 TEOS, 继续剧烈摚 拌, $1 \mathrm{~h}$ 后即得到起始溶胶.

分别将水填充的支撑体、干燥支撑体放入反应釜, 加入 $50 \mathrm{~mL}$ 溶胶, 密封, $100^{\circ} \mathrm{C}$ 反应 $72 \mathrm{~h}$ 后取出, 用去 离子水冲洗膜表面, 放入盛有 $\mathrm{EtOH} / \mathrm{HCl}(\mathrm{pH}=0)$ 溶液 的烧杯中, 在恒温水浴摇床上 $40^{\circ} \mathrm{C}$ 三级萃取 $(36 \mathrm{~h})$ 以 去除表面活性剂, 然后取出用去离子水冲洗, $120^{\circ} \mathrm{C}$ 干燥 $24 \mathrm{~h}$, 得到一次合成 MCM-48 膜. 二次合成与一 次合成步骤类似, 即将一次合成的膜用溶液填充后, 放入溶胶中水热合成, 经后续处理后得到二次合成 MCM-48 膜.

$X$ 射线粉末衍射(XRD)在 Rigaku D/Max-RA X 射 线衍射仪上进行, 采用 $\mathrm{Cu}$ K $\alpha$ 射线; BET 在 Micrometics ASAP-2020 型测定仪上进行, 采用液氮温度 $(77 \mathrm{~K})$ 下 的氮气吸附数据求得 BET 比表面积, 平均孔径根据 BJH 模型计算; SEM 在 LEO 1530 VP 扫描电镜上进行, 能谱在 INCAx-sight 能谱仪上进行.

\section{2 结果与讨论}

\subsection{MCM-48 粉体表征}

在合成 MCM-48 分子篮膜的同时, 反应釜内会 产生大量的 MCM-48 分子筛. 分子篎经 $\mathrm{EtOH} / \mathrm{HCl}$ 萃 取后干燥, 进行小角度 XRD 衍射表征(如图 1). 从图 1 可以看出, 水热合成 $72 \mathrm{~h}$ 能够得到晶型完整的 MCM-48 分子篮, 其特征峰显著.

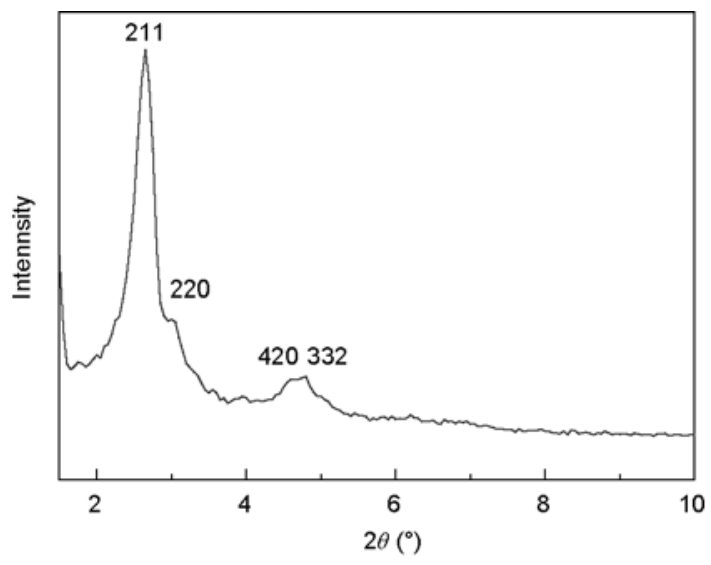

图 1 MCM-48 分子篮粉体 XRD 图

MCM-48 粉体的 $\mathrm{N}_{2}$ 吸附平衡等温线和孔径分布 曲线如图 2. 由图 2 可知, 样品呈现典型的 IV 型吸附 平衡等温线, 没有迟滞现象, 在 $P / P_{0}$ 为 $0.2 \sim 0.3$ 范围 内等温线有明显的突越，表明样品的孔径分布均一， 经 BJH 计算可得最可几孔径为 $2.38 \mathrm{~nm}$, BET 比表面 积为 $842.3 \mathrm{~m}^{2} / \mathrm{g}$, 与文献[3]报道的一致.

\subsection{SEM与能谱图表征MCM-48 膜}

干燥支撑体一次合成 MCM-48 膜表面存在着明 显的开裂现象如图 3(a), 水填充一次合成的 MCM48 膜表面没有大的缺陷如图 3(c). 对比图 3(a)和(c) 可以看出两种膜表面形态大有不同, 从放大倍数为 40000 倍的照片图 3(b)上看, 水填充支撑制备得到的 膜为球形颗粒状, 颗粒与颗粒之间紧密连接. 这与文 献[15]中利用乙醇作为溶剂室温下合成 MCM-48 分 子篮颗粒形貌基本上是一致的. 干燥支撑体和水

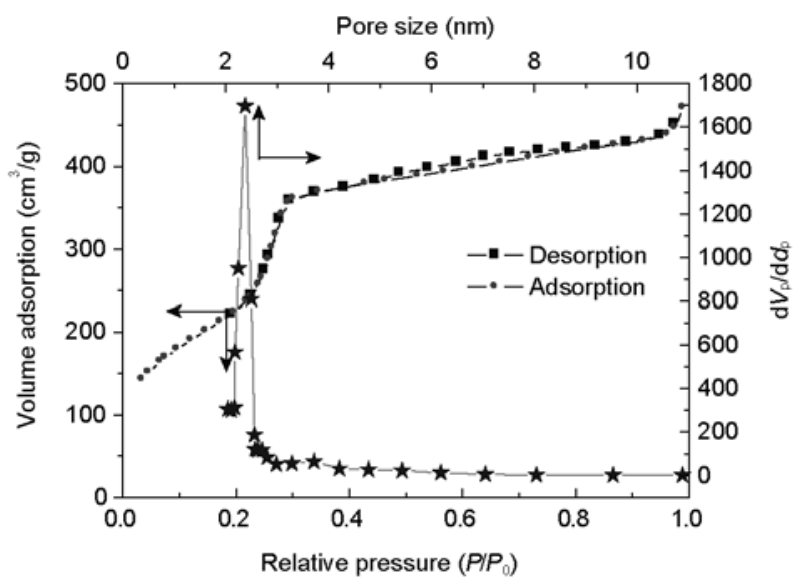

图 $2 \mathrm{MCM}-48$ 分子耖 $\mathrm{N}_{2}$ 吸附平衡等温线与孔径分布曲线 

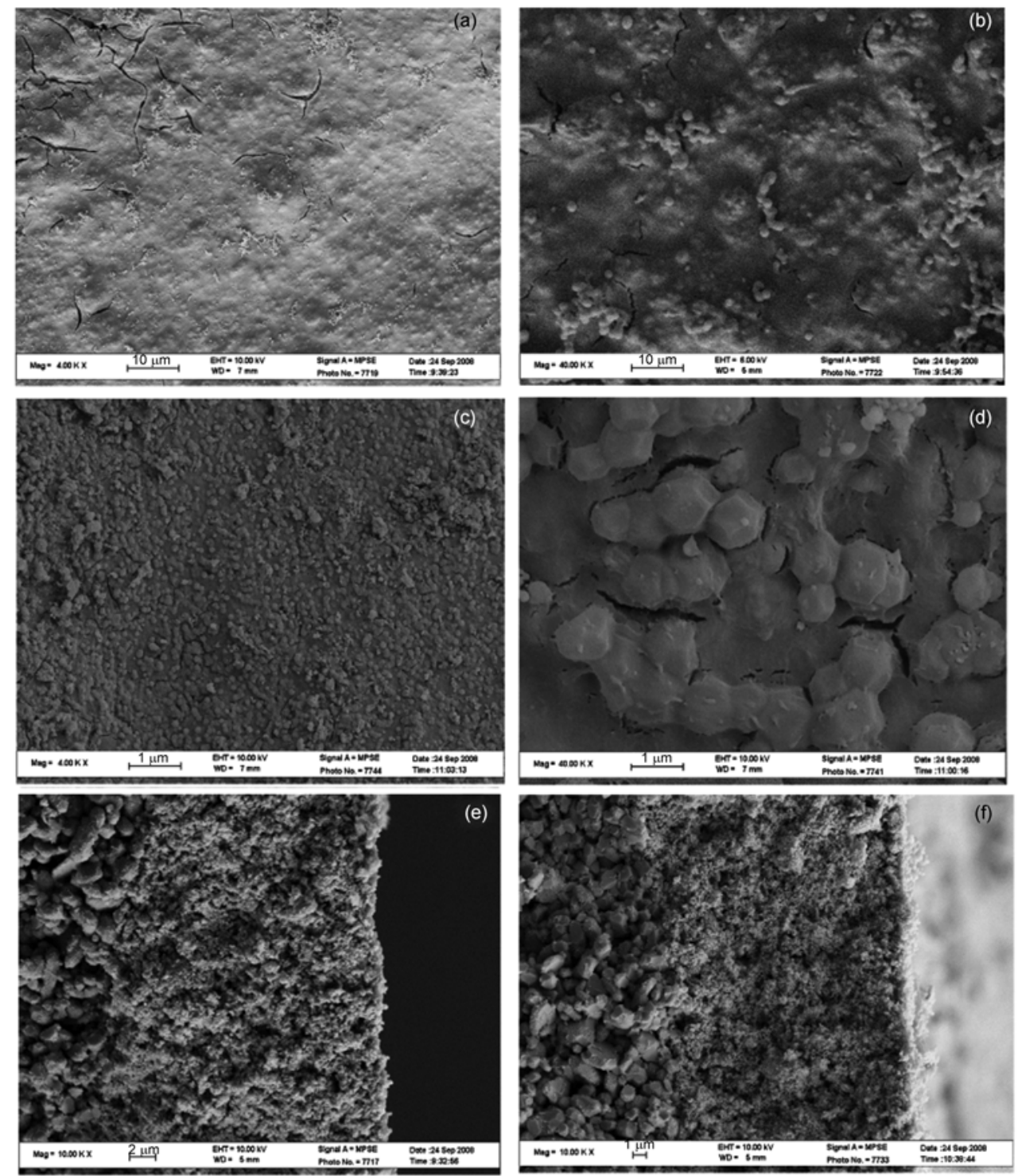

图 3 MCM-48 膜电镜图

(a) (b) 干燥支撑体一次合成膜表面; (c) (d) $\mathrm{H}_{2} \mathrm{O}$ 浸泡支撑体一次合成膜表面; (e) 干燥支撑体合成膜断面; (f) $\mathrm{H}_{2} \mathrm{O}$ 浸泡支撑体合成膜断面图

填充支撑体合成 MCM-48 膜断面照片如图 3(e)和(f), 支撑体表面都能够形成连续的膜层，厚度小于 $1 \mu \mathrm{m}$, 膜层与支撑体结合紧密.

在干燥支撑体放入溶胶的过程中存在着毛细吸 浆作用，这会导致溶胶渗入支撑体内部. 通过对水填 充支撑体、干燥支撑体合成 MCM-48 膜断面支撑层 采用线扫描能谱分析(图 4 和 5)可以看出, 水填充支 撑体合成的 MCM-48 膜时支撑体顶层基本没有渗透, 元素含量分析未发现支撑体中 $\mathrm{Si}$ 元素存在. 干燥支
撑体合成的 MCM-48 膜时支撑体顶层存在着部分溶 胶的渗透现象, 支撑体顶层中 $\mathrm{Si}$ 元素原子百分比为 $3.5 \%$. 可见水填充支撑体能够有效地防止溶胶的渗 透, 从而减小膜的透过阻力.

\section{3 气体渗透实验}

不同条件下合成膜的单气体渗透系数及 $\mathrm{H}_{2} / \mathrm{N}_{2}$ 分 离因子如图 6 所示. 从图 6 可以看出, 水填充支撑体 一次合成 MCM-48 膜 $\mathrm{b}$ 与干燥支撑体一次成膜 $\mathrm{a}$ 相 


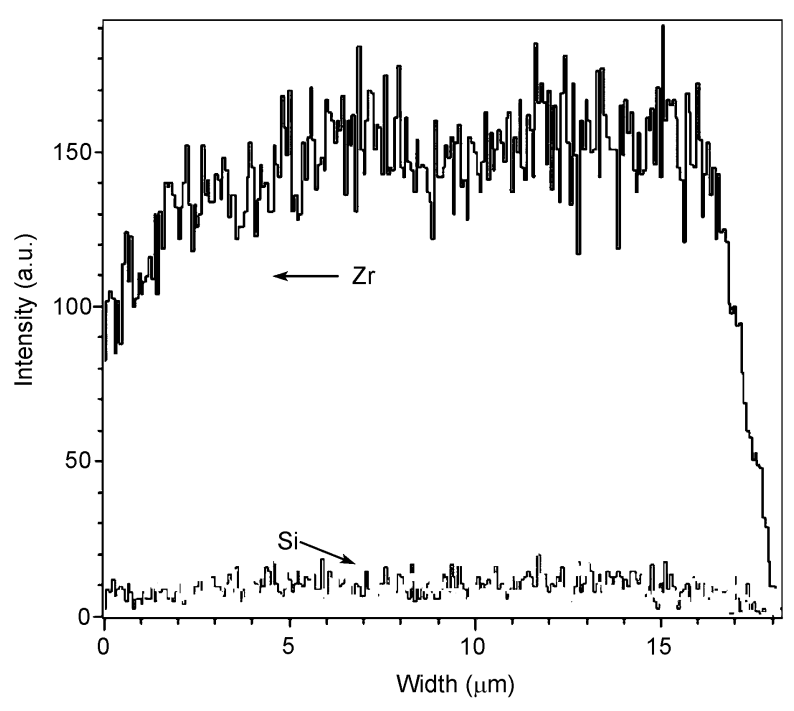

图 $4 \mathrm{H}_{2} \mathrm{O}$ 填充支撑体制备 MCM-48 膜断面线扫面能谱图

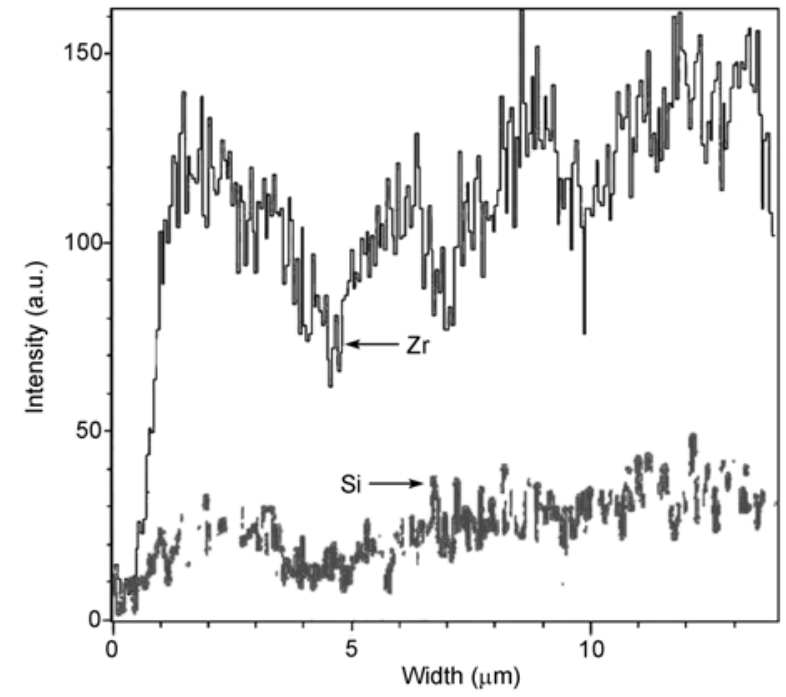

图 5 干燥支撑体制备 MCM-48 膜断面线扫面能谱图
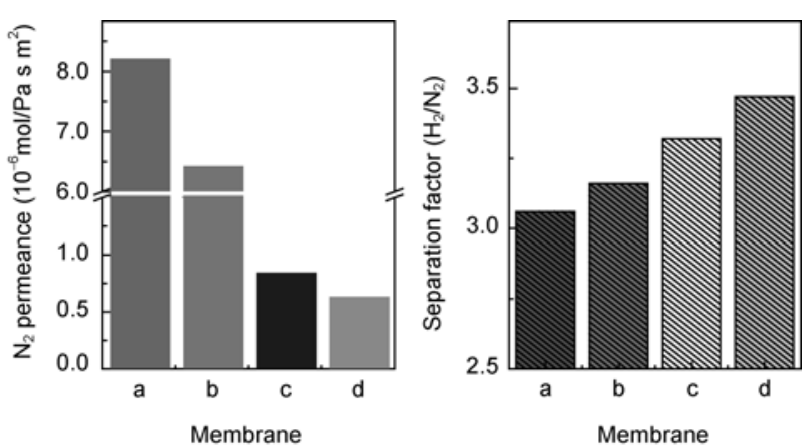

图 6 气体渗透系数与分离因子 (a 为干燥支撑体一次合成 膜; $b$ 为 $\mathrm{H}_{2} \mathrm{O}$ 填充一次合成膜; $\mathrm{c}$ 为干燥支撑体二次合成; d 为 $\mathrm{H}_{2} \mathrm{O}$ 填充二次合成膜)
比, 气体渗透系数略微下降, 分离因子提高. 结合 SEM 照片分析, 膜 a 表面存在着明显的开裂, 这是导 致其渗透系数较大, 分离因子较低的主要原因. 分别 通过二次合成得到干燥二次合成 MCM-48 膜 $\mathrm{c}$ 和水 填充二次合成 MCM-48 膜 $\mathrm{d}$, 二次合成与一次合成相 比, 气体渗透通量减小, 分离因子增大并接近努森扩 散理想分离因子 3.74, 膜完整性提高. 在常温、跨膜压 差 $0.05 \mathrm{MPa}$ 下膜 $\mathrm{d}$ 的 $\mathrm{N}_{2}$ 渗透系数为 $5.66 \times 10^{-7} \mathrm{~mol} /$ $\left(\mathrm{Pa} \cdot \mathrm{s} \cdot \mathrm{m}^{2}\right), \mathrm{H}_{2} / \mathrm{N}_{2}$ 分离因子可达到 3.47. 可见水填充支 撑体与干燥支撑体相比 MCM-48 膜完整性提高, 二 次合成有利于 MCM-48 膜完整性的提高.

水填充支撑体一次合成、二次合成 MCM-48 膜 $\mathrm{N}_{2}$ 和 $\mathrm{H}_{2}$ 渗透系数随压差变化如图 7 所示, 从图 7 可 以看出一次合成 $\mathrm{H}_{2}$ 和 $\mathrm{N}_{2}$ 渗透系数较高, 随着跨膜压 差的增大而增大，不是理想的努森扩散。二次合成 MCM-48 膜气体渗透系数基本不随跨膜压差的增大 而变化，其扩散机制为努森扩散.

Aksay 等人 ${ }^{[16]}$ 在对分子笁在固-液界面的形成过 程的研究中认为, 该过程是一个异相聚合过程, 模板 剂在不同的基体表面以不同的结构聚集，从而导致 膜层不同孔结构的形成. Gaub 等人 ${ }^{[17]}$ 也证实了模板 剂在不同极性的固体表面形成不同的表面活性剂胶 束. 据此可以推断, 支撑体表面的性质会影响表面活 性剂在其表面的形成状态, 最终影响膜层结构. 水填 充支撑体使 $\mathrm{ZrO}_{2}$ 支撑体表面性质更加接近溶胶, 在 支撑体表面形成的 MCM-48 膜与 MCM-48 分子笁的 形态基本一致, 这表明水填充有利于 MCM-48 分子 篮在支撑体表面形成完美的晶型、完整的膜层结构.

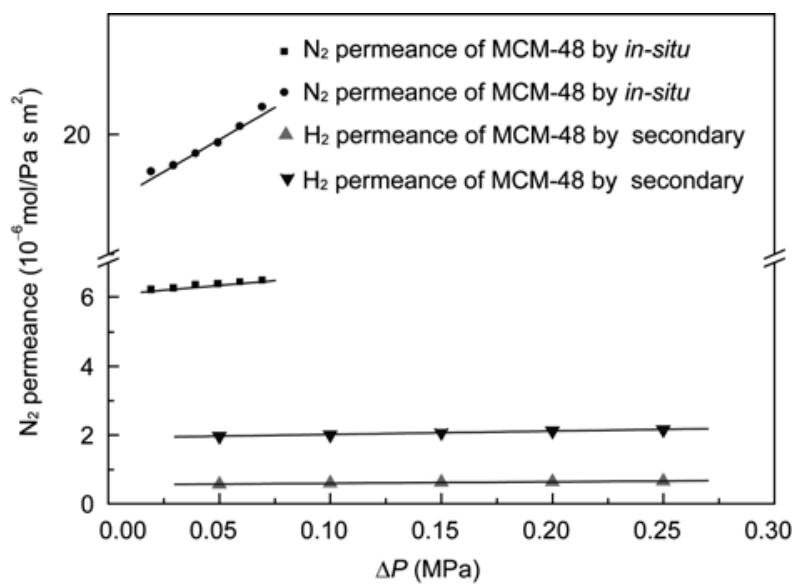

图 7 预填充制备MCM-48 膜 $\mathrm{N}_{2}$ 渗透系数随跨膜压差变化 


\section{3 结论}

采用 $\mathrm{ZrO}_{2}$ 管式非对称支撑体制备得到 MCM-48 膜, 大大减小了 MCM-48 膜的物质通过阻力, 有利于 提高 MCM-48 分子篮膜的气体渗透系数. 利用水填 充法可以防止毛细吸浆作用的产生, 进而减少溶胶 向支撑体内部的渗透. 利用水作为填充物不会残留 在支撑体之中, 不会造成额外的渗透阻力. 水填充支
撑体可以使支撑体表面更加接近溶胶，因此不会影 响 MCM-48 分子篎的合成, 有利于 MCM-48 分子篮 在支撑体表面形成均一的膜层, 减少合成次数. 通过 二次合成可以得到完整的、高通量的 MCM-48 分子 笁膜, 常温、跨膜压差为 $0.05 \mathrm{MPa}$ 下 $\mathrm{N}_{2}$ 平均渗透系 数为 $5.66 \times 10^{-7} \mathrm{~mol} /\left(\mathrm{Pa} \cdot \mathrm{s} \cdot \mathrm{m}^{2}\right), \mathrm{H}_{2} / \mathrm{N}_{2}$ 分离因子可达到 3.47 , 其渗透系数基本不随跨膜压差的变化而变化, 其扩散机理为努森扩散.

\section{参考文献}

1 Beck J S, Vartuli J C, Roth, W J, et al. A new family of mesoporous molecular sieves prepared with liquid crystal templates. J Am Chem Soc, 1992, 114(27): 10834-10843

2 Kresge C T, Leonowicz M E, Roth W J, et al. Ordered mesoporous molecular sieves synthesized by a liquidcrystal template mechanism. Nature, 1992, 359(6397): 710-712

3 Schumacher K, Ravikovitch P I, Du C A, et al. Characterization of MCM-48 materials. Langmuir, 2000, 16(10): 4648 - 4654

4 Alfredsson V, Anderson M W. Structure of MCM-48 revealed by transmission electron microscopy. Chem Mater, 1996, 8(5): 1141-1146

5 Nishiyama N, Koide A, Egashira Y, et al. Mesoporous MCM-48 membrane synthesized on a porous stainless steel support. Chem Commun, 1998, (19): 2147-2148

6 Nishiyama N, Park D H, Koide A, et al. A mesoporous silica (MCM-48) membrane: Preparation and characterization. J Membr Sci, 2001, 182(1-2): 235-244

7 Huang L, Kawi S, Hidajat K, et al. Preparation of M41S family mesoporous silica thin films on porous oxides. Microporous Mesoporous Mater, 2005, 82(1-2): 87—97

8 de la Iglesia O, Pedernera M, Mallada R, et al. Synthesis and characterization of MCM-48 tubular membranes. J Membr Sci, 2006, 280(1-2): $867-875$

9 Kumar P, Ida J, Kim S, et al. Ordered mesoporous membranes: Effects of support and surfactant removal conditions on membrane quality. J Membr Sci, 2006, 279(1-2): 539-547

10 Kumar P, Ida J, Guliants V V. High flux mesoporous MCM-48 membranes: Effects of support and synthesis conditions on membrane permeance and quality. Microporous Mesoporous Mater, 2008, 110(2-3): 595—599.

11 Kim Y S, Yang S M. Preparation of continuous mesoporous silica thin film on a porous tube. Adv Mater, 2002, 14(15): 1078-1081

12 Liu C, Wang J, Rong Z. Mesoporous MCM-48 silica membrane synthesized on a large-pore alpha- $\mathrm{Al}_{2} \mathrm{O}_{3}$ ceramic tube. J Membr Sci, 2007, 287(26): $6-8$

13 Liu C, Wang L, Ren W, et al. Synthesis and characterization of a mesoporous silica (MCM-48) membrane on a large-pore $\alpha-\mathrm{Al}_{2} \mathrm{O}_{3}$ ceramic tube. Microporous Mesoporous Mater, 2007, 106(1-3): 35-39

14 Ji H, Fan Y, Jin W, et al. Synthesis of Si-MCM-48 membrane by solvent extraction of the surfactant template. J Non-Crystal Solids, 2008, 354(18): $2010-2016$

15 Schumacher K, Grün M, Unger K K, et al. Novel synthesis of spherical MCM-48. Microporous Mesoporous Mater, 1999, 27(2-3): 201206

16 Aksay I A, Trau M, Manne S, et al. Biomimetic pathways for assembling inorganic thin films. Science, 1996, 273(5277): 892-898

17 Manne S, Gaub H E. Molecular organization of surfactants at solid-liquid interfaces. Science, 1995, 270(5242): 1480—1482 